\title{
Evaluation of Pre-operative Dermoscopy in Early Diagnosis of Non-melanocytic Skin Cancer
}

\author{
Reihane Bislimi Berisha ${ }^{1 *(D)}$, Djordje Dzokic ${ }^{2}$, Shkendije Dobruna $^{3}$ \\ ${ }^{1}$ Department of Dermatology, University Clinical Centre of Kosovo, Pristina, Kosovo; ${ }^{2}$ Department of Plastic Surgery, University \\ Clinical Centre of Skopje, Republic of Macedonia, ${ }^{3}$ Department of Dermatology, Private Clinical DermaD, Pristina, Kosovo
}

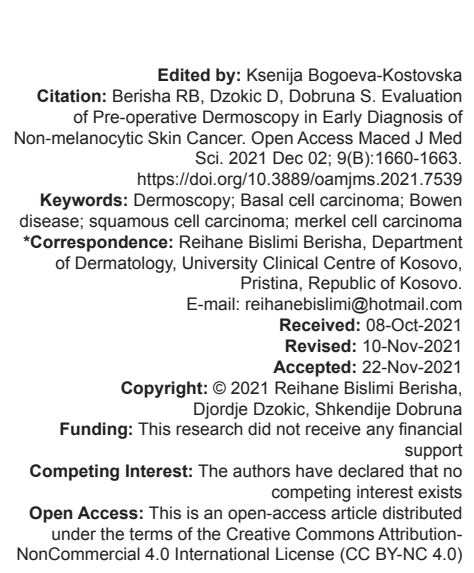

\section{Introduction}

Dermoscopy (also called epiluminescence microscopy, epiluminoscopy, and dermatoscopy) is a magnification and non-invasive, in vivo technique used for the evaluation of pigmented lesions of the skin [1], [2], [3].

With a dermoscope it is possible to visualize the structure, we not see with the naked eye by demonstrating significant additional morphological details. These structures are called epidermis, dermoepidermal junction, papillary dermis, and reticular dermis. These morphological structures (dermoscopy) associated with the correlation cutaneous histopathology.

By knowing the dermoscopy structures and their histopathological equivalents, the examiner will be able to distinguish between melanocytic and non-melanocytic and benign and malignant lesions, and thus between melanoma and non-melanoma which is in fact the main function of both diagnostic methods. This method has a great benefit in differentiating non-melanocytic from melanocyte lesions, differentiation between melanoma and non-melanoma lesions, reveals criteria that by pattern analysis yield give the correct diagnosis [4].

It also avoids unnecessary excisions and allows follow-up of patients with multiple nevi [5].

Dermoscopy is an important diagnostic tool that serves as a bridge to link clinical dermatological observation and histopathology findings. This is demonstrated by enhanced accurate diagnosis in different skin tumors. Herein, we include the most relevant dermoscopically structures in both melanocytic and non-melanocytic lesions and their precise histopathological correlation [6]. Dermoscopy is a fast imaging technique that allows an excellent visualization of non-melanocytic skin cancer (NMSC) [7].

\section{Technique of performing dermoscopy}

The technique of performing dermoscopy is very simple, fast, and cheap. It is a non-invasive 
method, avoided in vivo, so that the device itself leans toward the lesion to be seen. May be used a liquid medium (gel, oil, and alcohol) which improves the contact of the dermoscope with the lesion and thereby reduces the reflection. This technique refers to simple dermoscopy, digital and video dermoscopy is used for permanent recording and photo documentation [6]. The handheld dermoscope itself is an optical device that uses a magnifying glass, usually with (10.20 times magnification) using a halogen light source.

There are two types of dermoscopy, non-polarized, and polarized dermoscopy [8].

Non-polarized dermoscopy uses magnifying lenses and LED illumination light. This method requires contact with pre-liquid (gel, oil, and alcohol) skin to reduce reflection. Non-polarized dermoscopy allows visualization of structures located in the epidermis and dermoepidermal junction, but not below it.

Polarized dermoscopy in addition to the magnifying and light lenses, it uses two polarizing filters to enable cross-polarization. This type of method does not require liquid medium on the skin surface and also does not require skin contact. Polarized dermoscopy allows visualization of structures located deeper and below the dermoepidermal junction and the superficial dermis.

The main difference between non-polarized and polarized dermoscopy is the depth of visualization in the skin, non-polarized dermoscopy is good for visualization of structures located in the epidermis and dermoepidermal junction, as opposed to polarized which is betterfor visualizing deeper structures under dermoepidermal junction and the superficial dermis. Furthermore, for visualization of vascular structure is preferred polarized dermoscopy [7], [8].

\section{Dermoscopy diagnostic primary algorithm}

Dermoscopy methods have been developed to aid in the differential diagnosis of skin cancer. The most widespread is "two-step algorithm dermoscopy" [4]. First step - to differentiate melanocytic from no melanocytic lesions [6]. The dermoscopy should classify the lesion as melanocyte or non-melanocyte based on the observed dermoscopically structure and elements. Typical melanocyte lesion structures are (pigment lattice, globular, parallel structures, and strips). When the lesion does not have any positive element for melanocyte lesion, but does not meet the criterion for non-melanocyte, it should be considered in the context of atypical melanocyte lesion. Second step - is to distinguish benign melanocytic lesions from malignant melanoma using one of the following methods (Pattern analysis, $A B C D$ rule, Menzies method, and 7-point rule).

\section{NMSC}

Skin cancer is one of the most common cancers in the world. NMSC is the most common form of cancers that slowly develop in the upper layers of the skin [10]. Based on the WHO classification for skin tumors: Keratinocytic/epidermal (non-melanoma): Basal cell carcinoma (BCC), squamous cell carcinoma (SCC), SCC in situ (Bowen disease), and Merkel cell carcinoma (MCC).

\section{$B C C$}

Is the most common type of skin cancer and the most common neoplasm in humans [11]. BCC accounts for $75 \%$ of cases and is a slow-growing, locally invasive epidermal tumor with a metastatic rate of $<0.1 \%$ [10].

Clinically, there are different types: Nodular-ulcerative, pigmented, diffuse (infiltrating and morpheaform), fibroepithelial, and superficial.

\section{SCC}

SCC appears as a firm pink lump with a rough or crusted surface. There can be a lot of surface scale and sometimes even a spiky horn sticking up from the surface.

The lump often feels tender when touched, bleeds easily, and may develop into an ulcer.

\section{Bowen disease (SCC in situ)}

Is a precancerous form of SCC? It develops slowly and is easily treated. The main sign is a red, scaly patch on the skin that may itch. It most commonly affects elderly women and is often found on the lower leg. However, it can appear on any area of skin.

\section{MCC}

MCC is historically classified among neuroendocrine tumors, although its behavior resembles, in the most instances, $\mathrm{CM}$ for the high propensity to colonize lymph nodes [11], [12].

\section{Diagnosis of NMSC}

Diagnosis of NMSC is usually dermoscopy and clinical. Dermoscopy is a widely accessible, cheap, and reliable non-invasive diagnostic tool that improves the accuracy of the clinical diagnosis of NMSC. Dermoscopy provides a more complete and detailed diagnosis of NMSC. To make a diagnosis, it is necessary to do a dermoscopy of the full body, to take a good history [13].

\section{Dermoscopy characteristics of NMSC}

Dermoscopy remarkably enhanced diagnostic accuracy, skin cancer detection sensitivity, and confidence in diagnosis of NMSC [14]. Dermoscopy 
is a fast imaging technique that allows an excellent visualization of NMSC [7].

The dermoscopically criteria associated with BCC include the absence of a pigment network and the presence of specific features, for example, arborizing vessels, large blue-gray ovoid nests, multiple blue-gray globules, leaf-like areas, spoke wheel areas, and ulceration.

Dermoscopic criteria for SCC include the presence of keratin/scales, blood spots, white circles, white structure less areas, hairpin vessels, linear-irregular vessels perivascular white halos, and ulceration.

The dermoscopy characteristics of Bowen's disease (BD) - glomerular vessels (69-97\%) and scaly white-to-yellow surfaces (64-96\%) were commonly observed in non-pigmented BD, brown-to-gray globules/dots and structure less pigmentation were observed in 21-80\% and 70-78\% of pigmented BD [14].

The dermoscopy characteristics of MCC finding are the milky-red areas that are usually associated with linear irregular vessels and structure less areas [15], [16].

\section{Aim}

The main aim of the study is to prove the value of dermoscopy in the precision of pre-operative diagnosis of NMSC confirmed by post-operative $\mathrm{PH}$ findings.

Additional goals are to declare dermoscopy subtypes of NMSC in according to the age, sex, localization, UV radiation, anatomical region, and phototype of skin.

\section{Materials and Methods}

To carry out, this research was being obtained at the Department of Dermatology-University Clinical Centre of Kosovo, Institute of Pathology University of Kosovo.

Retro-prospective study will be applied.

In this research, a total of 50 patients were included in this study, and a total of 11 of them were analyzed during my 3 years of experience in the dermatological oncology clinic at the dermatovenereology clinic.

Where included in the study, 11 patients with nine histopathologically $(\mathrm{PH})$ proved NMSC.

Parameters of follow-up for grouping patients will be gender, age, localization, profession, and NMSC histological subtype.

Inclusion criteria were a definite histopathologic diagnosis of NMSC, including subtype classification, the availability of clinical, dermatoscopic, and the availability of histopathologic slides.

Theexclusioncriteriawere:Immunosuppressive therapy, hematological diseases (e.g., leukemia and lymphoma), and hemophilia, and the presence of extensive ulceration was excluded from the study.

A complete dermoscopically examination of the whole body will be performed by dermatoscopic images were captured with Dermlite Foto equipment (Dermlite 3Gen, Heine) at ten-fold magnification. A digital recording is done with apple iPhone of dermoscopically images.

Cases with complete wide incision, as well as histopathological $(\mathrm{PH})$ examination were a result of punch biopsy or shave biopsy. $\mathrm{PH}$ weekly was applied to them to confirm the diagnosis given by the dermatoscopy examination.

\section{Results}

A total of 11 cases were collected, with nine histopathologically $(\mathrm{PH})$ proven of NMSC.

The median age of patients was 72 years $(S D \pm 15.15)$ and 6 of $10(60 \%)$ were female. The lesions were located mainly on the face with predilection nose (six cases, 60\%), followed by back (two cases, 20\%), and three cases (30\%) on other body sites (foot, scalp, and trunk).

The NMSC histological subtype was: Seven cases (70\%) BCC and two cases (20\%) SCC.

Patients were asked a set of questions about their sun exposure habits. The job of $3(30 \%)$ respondents is about sun exposure, with the most of the work exposed for $<3 \mathrm{~h}$. In addition, 5 (50\%) respondents were exposed for more than $6 \mathrm{~h}$. The most respondents spend 15-30 days in the sun on annual vacation (on a mountain, lake, or sun).

The results of this study provided important information regarding the use of SPF funds in our population. Namely, out of five respondents, they used SPF only during summer vacation, one of the respondents used SPF continuously, unlike four who never used SPF. None of them had ever used artificial tanning springs.

Patients were analyzed for their skin according to the Fitzpatrick scale. According to the results, this group of respondents was dominated by three phototypes: Phototypes II, III, and IV.

During my 3 year practice in dermatopathology, ambulatory internship I came to the conclusion that the viewed and operated patients are confirmed by $\mathrm{PH}$ findings. 
From finding $\mathrm{PH}$ in favor of accuracy pre-operative dermoscopy, it is evidence proven that dermoscopy accuracy and early diagnosis of NMSC compared with the unaided eye.

Hence, the results of our pre-operative dermoscopy are of great value in precision of pre-operative diagnosis which being proved by post-operative $\mathrm{PH}$ findings.

\section{Conclusion}

Our first experiences with pre-operative dermoscopy in 11 patients indicate its value in the diagnosis of NMCS. Our further studies in multiple patients should determine its accuracy in pre-operative diagnosis confirmed by post-operative $\mathrm{PH}$ findings.

\section{References}

1. Marghoob AA, Swindle LD, Moricz CZ, Negron FA, Slue B, Halpern $A C$, et al. Instruments and new technologies for the in vivo diagnosis of melanoma. J Am Acad Dermatol. 2003;49(5):777. http://doi.org/10.1016/s0190-9622(03)02470-8 PMid: 14576657

2. Menzies SW, Ingvar C, McCarthy WH. A sensitivity and specificity analysis of the surface microscopy features of invasive melanoma. Melanoma Res. 1996;6(1):55. http://doi.org/10.1097/00008390-199602000-00008 PMid:8640071

3. Argenziano G, Soyer HP. Dermoscopy of pigmented skin lesions--a valuable tool for early diagnosis of melanoma. Lancet Oncol 2001;2(7):443. http://doi.org/10.1016/ s1470-2045(00)00422-8 PMid:11905739

4. Rabinovitz S, Oliviero M, Kopf AW, Saurat JH. Dermoscopy of pigmented skin lesions. JAmAcad Dermatol. 2005;52(1):109-21. http://doi.org/10.1016/j.jaad.2001.11.001 PMid:15627088

5. Friedman RJ, Rigel DS, Silverman MK, Kopf AW, Vossaert KA. Malignant melanoma in the 1990s: The continued importance of early detection and the role of physician examination and self-examination of the skin. CA
Cancer J Clin. 1991;41(4):201-26. http://doi.org/10.3322/ canjclin.41.4.201

\section{PMid:2049635}

6. Pehamberger $\mathrm{H}$, Binder $\mathrm{M}$, Steiner $\mathrm{A}$, Wolff $\mathrm{K}$. In vivo epiluminescence microscopy: Improvement of early diagnosis of melanoma. J Invest Dermatol. 1993;100(3):356S-362S http://doi.org/10.1016/s0190-9622(87)70240-0

PMid:3668003

7. Stolz W, Braun-Falco O, Bilek P, Landthaler M, Cognetta AB. Color Atlas of Dermatoscopy. Vol. 24. Oxford: Blackwell Science Ltd.; 1994. p. 69-72.

8. Chen LL, Dusza SW, Jaimes N, Marghoob AA. Performance of the first step of the 2-step dermoscopy algorithm. JAMA Dermatol. 2015;151(7):715-21. http://doi.org/10.1001/ jamadermatol.2014.4642

PMid:25714607

9. Samarasinghe V, Madan V. Nonmelanoma skin cancer. J Cutan Aesthet Surg. 2012;5(1):3-10. http://doi. org/10.4103/0974-2077.94323 PMid:22557848

10. Palacios-Martínez D. Dermoscopy for beginners (ii): Dermoscopic structures and diagnostic methods. Semergen. 2017;43(4):312-7. http://doi.org/10.1016/j.semerg.2015.11.010

PMid:26775539

11. Newlands C, Currie R, Memon A, Whitaker S, Woolford T. Non-melanoma skin cancer: United Kingdom national multidisciplinaryguidelines. JLaryngolOtol.2016;130(2):S125-32. http://doi.org/10.1017/S0022215116000554 PMid:27841126

12. Urrego-Rivera FL, Faura-Berruga C. Differential diagnosis of pigmented basal cell carcinoma. Rev Clin Med Fam. 2015;8(2):166-70.

13. Kato J, Sato S, Minowa T, Uhara H. Dermoscopy of melanoma and non-melanoma skin cancers. Front Med. 2019;6:180. http://doi.org/10.3389/fmed.2019.00180 PMid:31497603

14. Cives M, Mannavola F, Lospalluti L, Sergi MC, Cazzato G, Filoni $\mathrm{E}$, et al. Non-melanoma skin cancers: Biological and clinical features. Int J Mol Sci. 2020;21(15):5394. http://doi. org/10.3390/ijms21155394

PMid:32751327

15. Fargnoli MC, Kostaki D, Piccioni A, Micantonio T, Peris $\mathrm{K}$ Dermoscopy in the diagnosis and management of non-melanoma skin cancers. Eur J Dermato. 2012;22(4):456-63. http://doi.org/10.1684/ejd.2012.1727 PMid:22534287

16. Longo $C$, Lallas A, Kyrgidis A, Rabinovitz $H$, Moscarella $E$, Ciardo $A$, et al. Classifying distinct basal cell carcinoma subtype by means of dermatoscopy and reflectance confocal microscopy. J Am Acad Dermatol. 2014;71(4):716-24.e1. http:// doi.org/10.1016/j.jaad.2014.04.067

PMid:24928707 